\title{
A Rare Case of a Large Meningocele Posterior Sacrococcygeal
}

\author{
Rakotondraibe Willy Francis, Bemora Joseph Synèse, Habib Nouraly, Raveloharimino Nirina \\ Henintsoa, Tsiaremby Marc Guichard, Rabesandratana Norotiana, Andrianarimanana Diavolana, and \\ Andriamamonjy Clément
}

\section{ABSTRACT}

We report a case of sacrococcygeal meningocele simulating a sacrococcygeal teratoma. Likewise, there are a discussion of the pathophysiology and surgical approach of these types of lesions. We present a case of an exceptionally large posterior coccygeal meningocele of a newborn, and show the interest of radio-echographic couple and the interest of a management surgical approach. This was a case of posterior coccygeal meningocele, in a 10-day-old newborn born at term, with no known maternal history other than active smoking. The clinical examination finds a eupneic newborn, presents a median mass at the level of the sacrococcygeal part, of soft consistency, painless, without signs of inflammation, of normal skin, volume $18 \times 21 \times 19 \mathrm{~cm}$, with positive transillumination test, no sensitivomotor deficit of both limbs, no other malformative anomalies detected clinically, but presence of anal atony and vesico-anal inconstancy. The ultrasound reveals a fluid formation containing small cysts. The $x$-ray revealed the absence of the posterior coccyx arch, an appearance in favor of a posterior sacrococcygeal meningocele. The surgery consisted of a meningocele cure followed by a buttock plasty. The postoperative follow-up was simple, without infectious or metabolic complications. Standardization and improvement of accessibility to ultrasound and obstetric MRI could improve the antenatal diagnosis of this pathology in Madagascar.

Keywords: closed spinal bifida, méningocèle, surgery x-ray computed
Published Online: February 20, 2021

ISSN: $2736-5476$

DOI : 10.24018/ejclinicmed.2021.2.1.17

Rakotondraibe Willy Francis *

Department of neurosurgery, CHU P ZAGA Mahajanga, Madagascar

(e-mail : wrakotondraibe ${ }^{\circledR}$ gmail.com) Bemora Joseph Synèse

Department of neurosurgery, CHU-JRA Antananarivo, Madagascar

(e-mail: josbemora@yahoo.fr)

Habib Nouraly

Imaging, CHU Mahavoky Sud Mahajanga, Madagascar

Raveloharimino Nirina Henintsoa

CHU P ZAGA Mahajanga, Madagascar

Tsiaremby Marc Guichard

Department of neurosurgery CHU

Tanambao I Antsiranana, Madagascar

Rabesandratana Norotiana

Neonatology CHU P ZAGA Mahajanga, Madagascar

Andrianarimanana Diavolana

Neonatalogy CHU P ZAGA Mahajanga,

Madagascar

Andriamamonjy Clément

Department of neurosurgery, CHU-JRA

Antananarivo, Madagascar

*Corresponding Author

\section{OBSERVATION}

Meningocele is a hernia of the meninge alone, filled only with cerebrospinal fluid. The nervous elements are in their place at the bottom of the spinal canal and in principle are not malformed. The meningeal hernia protrudes through the musculo-aponeurotic and bone breach. It is responsible for a posterior median swelling. It is usually covered with healthy skin [1], [2]. This is the mild form of Spina Bifida Aperta (SBA) and it accounts for only 10 to $15 \%$ of SBA. To our knowledge, no case of coccygeal meningocele has been reported in Malagasy literature to date. We present a case of an exceptionally large posterior coccygeal meningocele of a newborn, and show the interest of radio-echographic couple and the interest of a management surgical approach.
History of the disease and physical examination: 10-dayold newborn born at term and Apgar 10 presents at birth a mid-sacrococcygeal swelling of soft consistency, without any other malformation. The birth is vaginally from smoking mother 4 pack-years, without taking folic acid before conception as well as during antenatal consultations. The mother has no particular antecedent apart from active smoking; taking a decoction like Fenugreek (Angamay) during pregnancy has been revealed in the mother. The clinical examination found a eupneic newborn, median mass at the level of the sacrococcygeal part, soft consistency, painless, without signs of inflammation, normal skin, volume $18 \times 21 \times 19 \mathrm{~cm}$ (Figure 01), with positive transillumination test, no sensitivomotor deficit of both limbs, with normal tonicity as well as trophicity, normal transits, without other malformative anomalies detected clinically, but presence of 
anal atony and vesico-anal inconstancy. The fontanelles are normotensive, and the psychomotor development is unremarkable. The fundus was normal, no bilateral papillary edema.

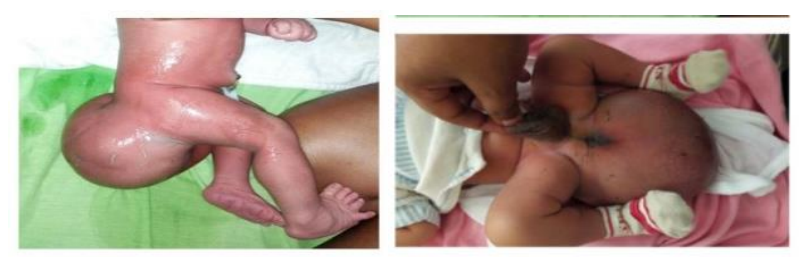

Fig. 1. large sacrococcygeal swelling with no other visible malformations

Imaging: Ultrasound of the newborn reveals fluid formation containing small cysts (Figure 2). No other malformations were individualized. Transfontanellar ultrasound finds normal ventricular size without parenchymal abnormalities or malformative abnormalities. Cardiac Doppler ultrasound, abdominal ultrasound and chest $\mathrm{x}$-ray found no other abnormalities or associated malformations. The X-ray was taken and the posterior coccyx arch was absent, this aspect is in favor of a sacrococcygeal meningocele (Figure 2).

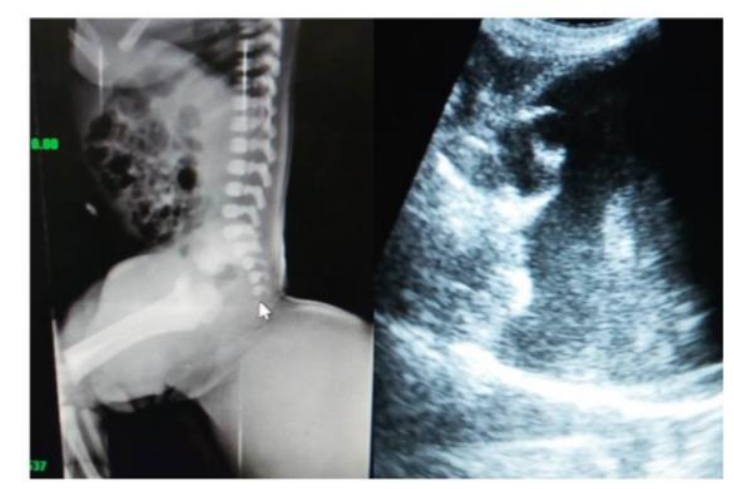

Fig. 2. X-ray and ultrasound showing a fluid coccygeal mass containing formations

Surgical technique: in view of the symptoms resulting from the mass effect of this swelling, coccygeal meningocele repair was performed with buttock plasty in the newborn. After an arcuate incision and detachment of the dura mater from the skin, $800 \mathrm{ml}$ of serohaematic cerebrospinal fluid was evacuated from the neurosurgical side. Subsequently, in the same act, a buttockplasty was performed by the plastic surgery team.

Histology: the pathological examination concluded in nervous tissue (meninge, peripheral nervous tissue), without cyto-nuclear atypia. There were no histologic signs of malignancy or obvious vascular abnormality. The diagnosis was therefore that of a coccygeal meningocele.

Postoperative evolution: the postoperative follow-up was simple, without infectious or metabolic complications. The scars were clean with no signs of inflammation. Anal tone was normal postoperatively and the newborn was passing stool without signs of faecal incontinence. He left home on D12 in front of a favorable evolution while keeping urinary incontinence. The patient was seen in an outpatient clinic for regular follow-ups and checks every 3 months, then 6 months and then every year until the age of 3 years. The urinary incontinence resolved within a month postoperatively, and he was in school at the age of 2.5 years, with no sphincter disturbances or signs of hydrocephalus.

\section{DISCUSSION}

SBA is a herniation of the dura mater and arachnoid material outside the spinal canal by a defect either in the posterior sacral wall, or posterolateral, by an intervertebral foramen, resulting in a generally cystic structure, filled with cerebrospinal fluid and, sometimes, neuronal elements and / or tissues, and is continuous with the spinal subarachnoid space [1], [3]-[6]. ASM can be congenital or acquired and in most cases the defect is a smooth oval structure with welldefined edges [9]. Coccygeal meningocele is a rare form of Spina Bifida Aperta (SBA). The incidence of this malformation among births in our country and its frequency in Madagascar are not established. Due to the lack of financial means of the patients, the diagnosis is often prenatal. The lack of prenatal consultation and obstetric ultrasound examination delay this delay in diagnosis. Epidemiological investigations show that the frequency of SBA depends on several factors [1], [4], [10]. Racially, this malformation is less common in blacks and yellows than in whites [11]. Exogenous factors influence the onset of this condition, particularly in patients from disadvantaged socio-professional categories in Great Britain [12]. These exogenous factors probably consist of a vitamin deficiency, in particular of folic acid [13]. This condition affects both genders. In our presentation, it is about a boy who gave birth to a smoking mother.

In acquired lesions, the proposed pathogenic mechanism consists of a pulsatile stress which dilates a predisposed dural tissue, which gradually develops resulting in bone erosion due to pressure fluctuations of the cerebrospinal fluid (CSL) [4], [5], [14], [15]. The highest pressures occur on the lower areas of the spinal canal, explaining the greatest impact on the sacral region [14].

It has also been described as the result of the elevation of SCL pressure caused by spontaneous SAH [16] leading to an enlargement of a pre-existing dural ectasia, characteristic of Marfan syndrome. This was the case reported by Strand in 1971, where the consistent clinical presentation of a subarachnoid hemorrhage occurred 2 months before diagnosis of a meningocele. However, in our case, the congenital explanation for probable folic acid deficiency (no FAF taken before conception) and the risk factor such as active smoking in the mother seem to us to be the cause of this mass.

Associated symptoms are generally rare and mild [17]. Postural headaches due to changes in intracranial pressure may result either from hydrostatic pressure directly on the cyst resulting from the retrograde transmitted Valsalva maneuver to the intracranial region or from hypotension due to position changes in adults [1], [8], [18]. In the newborn, the median swelling, of variable size, along the rachis is very characteristic; associated anomalies (hydrocephalus, Arnold Chiari malformation, Dandy Walker malformation, toe and / or finger malformation) are almost exclusive to SBAs, and all the more frequent the more severe and lower located [1], [10][12]. These lesions do not resolve spontaneously, and the risk of serious complications, meningitis [5], [15] leads to consider surgical intervention early on [1]. 
Imaging plays an important role in diagnosing a meningocele. An antenatal ultrasound allows early diagnosis and assesses the functional prognosis of the fetus. On the other hand, in postnatal care, magnetic resonance imaging remains a benchmark examination. But its availability and cost limit its prescription. In the city of Mahajanga, ultrasound due to its non-irradiating nature, supplemented by a standard x-ray, confirms the diagnosis and looks for other associated malformative anomalies. In our case no other congenital anomaly was detected. Carried out and interpreted by an experienced doctor, these two examinations show the dehiscence of the posterior vertebral arch and confirm the absence of nerve tissue in the meningeal pouch. The scanner is another exploration technique, but its irradiating nature limits its prescription in newborns.

The objectives of the surgery should be decompression of the spinal canal by drainage and / or excision of the mass, obliteration of the communication between the cyst and the subarachnoid space and resection of associated tumors, and detachment of the spinal cord, if necessary [5],[6]. The posterior transsacral approach, introduced by Adson in 1938, exposes communication with the meningocele. It consists of aspirating its contents through the pedicle and closing the communication with primary ligature or obliteration with a graft [3], [4], [7], [15], [16]-[18]. This is the approach most used by neurosurgeons and has traditionally been considered low risk and with fewer complications. It will always be the first choice when possible, as it allows better access to the pedicles and protection to the sacred roots, with lower risk of infection and morbidity, and it allows detachment when needed [2],[4], [9] . Secondly, the plastic surgery team performed a buttock plasty (Discharge incisions on both sides, allowing tension-free closure, plasty of the levator muscles, then of the buttocks muscles and possible drainage by a blade of Delbet then suture of the different planes). The use of endoscopy [5],[7],[18] has been recommended as an alternative, especially for non-obese patients with a small fistula connection.

We add a possible risk of postoperative infection and very rarely hemorrhagic because initially large (or if the intraoperative hemostasis was poor) hence the interest of an antibiotic therapy of good diffusion until postoperative D5 for the control clinical and regression of the lesion.

\section{CONCLUSION}

The spinal dysraphisme saccrococcygeal type meningocele is extremely rare, it sometimes breaks a diagnostic problem with teratomas, and the scanner makes it possible to make the diagnosis. The treatment is mainly surgical.

\section{CONFLICTS OF INTEREST}

All authors certify that they have no affiliation or involvement in any organization or entity with the interests (such as honoraria, scholarships, participation in speakers' offices; and expert testimony or agreements of patent license), or non-financial interests (such as personal or professional relationships, affiliations, knowledge or beliefs) in the subject or material discussed in this manuscript.

\section{REFERENCES}

[1] Beyazal M. An asymptomatic large anterior sacral meningocele in a patient with a history of gestation: a case report with radiological findings. Case Rep Radiol. 2013; 1-3.

[2] Anna AS, Morandi X. Anterior sacral meningocele in pregnancy. Case report. J Neurosurg 2001; 94:162-4.

[3] Khan AK, Deb S, Ray DK, Nag BK. Thecoperitoneal shunt in case of symptomatic anterior sacral meningocele. Neurol India 2004; 52:4034 .

[4] Marin-Sanabria EA, Nagashi T, Yamamoto K, Nakamura Y, Aihara H, Kohmura E. Presacral meningocele associated with hereditary sacral agenesis and treated surgically: evaluation in three members of the AME family. Neurosurgery 2004; 57: E597.

[5] Massimi L, Calisti A, Koutzoglou M, Di Roco C. Giant anterior sacral meningocele and posterior sacral approach. Childs Nerv Syst 2003;19:722-8.

[6] Sahin N, Genc M, Kasap E, Solak A, Korkut B, Yilmaz E. Anterior sacral meningocele masquerading as an ovarian cyst: a rare clinical presentation associated with Marfan syndrome. Clin Pract 2015; 5:446.

[7] Sunna TP, Westwick HJ, Zairi F, Berania I, Shedid D. Successful management of a giant anterior sacral meningocele with an endoscopic cutting stapler: case report. J Neurosurg Spine 2016; 24:862-6.

[8] Versteegh HP, Feitz WF, van Lindert EJ, Marcelis C, de Blaaw I. "This bicycle gives me a headache', a congenital anomaly. BMC Res Notes. 2013; 6: 412.

[9] M Thomas, Habaly FA, Hirschauer JS. Hereditary Occurrence of Anterior Sacral Meningocele: Report of Ten Cases. Spine 1987; 12(4), $351-4$.

[10] Frey L, Allen Hauser W. Epidemiology of Neural Tube Defects. Epilepsia, 2003; 44(3):4-13.

[11] Luben T. et al. " Epidemiology ». Urban/Rural Differences in Occurrence of Neural Tube Defects in Texas. 2008; 19 (6):102- 103.

[12] Au KS et al. «Dev Disabil Res Rev». Epidemiologic and genetic aspects of spina bifida and other neural tube defects.2010;16(1):6-15.

[13] Jennifer Williams et al. « CDC » Updated Estimates of Neural Tube Defects Prevented by Mandatory Folic Acid Fortification - United States, 1995-2011. 2015;64(01):1-5.

[14] Santana JM, Go'mez A, Aleman P, Travieso MM. Severe hydroureteronephrosis associated to asymptomatic giant anterior sacral meningocele: a case report and review of the literature. Radiologia 2007; 49:43-6.

[15] Sunna TP, Westwick HJ, Zairi F, Berania I, Shedid D. Successful management of a giant anterior sacral meningocele with an endoscopic cutting stapler: case report. J Neurosurg Spine 2016; 24:862-6.

[16] Raftopoulos C, Pierard GE, Rétif C, Braude P, Brotchi J. Endoscopic Cure of a Giant Sacral Meningocele Associated with Marfan's Syndrome. Neurosurgery, 1992; 30(5), 765-8

[17] Voyvodic F, Scroop R, Sanders RR. Anterior Sacral Meningocele as a Pelvic Complication of Marfan Syndrome. The Australian and New Zealand Journal of Obstetrics and Gynaecology, 1999; 39(2), 262-5.

[18] Stone JG, Bergmann LL, Takamori R, Donovan DJ. Giant pseudomeningocele causing urinary obstruction in a patient with Marfan syndrome. J Neurosurg Spine 2015; 23:77-80. R. A. Scholtz, "The Spread Spectrum Concept," in Multiple Access, N. Abramson, Ed. Piscataway, NJ: IEEE Press, 1993, ch. 3, pp. 121-3.

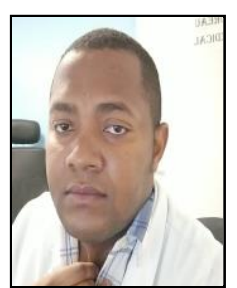

Rakotondraibe Willy is a neurosurgeon, head of neurosurgery at CHU P Zaga Mahajanga, he is also head of clinic in Neurosurgery. 